\title{
Innovations in Population Health Surveillance Using Electronic Health Record Data
}

\author{
Sharon E. Perlman*1, Katharine H. McVeigh ${ }^{1}$, Remle Newton-Dame ${ }^{1}$, Lorna E. Thorpe ${ }^{2}$, \\ Elisabeth F. Snell ${ }^{1}$, Claudia Chernov ${ }^{1}$, Jesse Singer ${ }^{1}$ and Carolyn M. Greene ${ }^{1}$
}

${ }^{1}$ New York City Department of Health and Mental Hygiene, Queens, NY, USA; ${ }^{2}$ City University of New York School of Public Health, New York, NY, USA

\section{Objective}

To describe the potential benefits and challenges of using electronic health record data for population health surveillance, and what to consider when establishing an electronic health record (EHR) surveillance system (EHRSS).

\section{Introduction}

EHRs are increasingly being adopted to improve quality of care in health care systems, but they also have potential to monitor health at the population level. There has been relatively little focus on using EHRs for population health surveillance beyond infectious diseases. Current tools to monitor population health - vital statistics, hospital discharge data, population health surveys - are useful but can be expensive, and may be slow to conduct or produce findings. Aggregated EHR-derived data have the potential to deliver cheaper and faster data, and have the capacity to provide information on earlier disease stages, thus increasing the likelihood of timely use. If EHR data can be validated, they can be used to augment existing surveillance methods, resulting in more strategic targeting of health resources and better data to guide and evaluate public health initiatives and policies. New York City (NYC) is currently developing a pilot public health surveillance program known as NYC Macroscope, the first domestic effort to aggregate EHR data from independent primary care practices into a surveillance tool. This EHR-based population health surveillance system will compile summarized data from ambulatory electronic health records to help city health officials monitor and respond to real-time data on conditions of public health importance.

\section{Methods}

We reviewed the literature and talked to national stakeholders at the federal, state, local levels in addition to clinical and academic experts. We have outlined key issues in developing an EHRSS, and developed proposed indicators for the NYC Macroscope. We will validate the proposed indictors by comparing them with data from a gold standard population health survey, NYC Health and Nutrition Examination Survey (HANES), currently being conducted.

\section{Results}

Proposed initial indicators for the NYC Macroscope include: prevalence, treatment and control of diabetes, high cholesterol and hypertension; depression prevalence; smoking status; body mass index; and influenza vaccination. Identified methodological challenges include: data structure and system design, data quality, length of observation, duplicate records, practice inclusion criteria, selection bias and generalizability of findings, and uncertainty when analyzing trends over time. Support from key public health leadership, negotiating legal obstacles to aggregating data and ensuring confidentiality are critical in developing an EHRSS.

\section{Conclusions}

EHR networks are rapidly being constructed in domestic and international settings. Important methodological and operational considerations identified through planning for the NYC Macroscope will inform others interested in using EHRs to monitor population health.

\section{Keywords}

surveillance; population health; electronic health records

\section{Acknowledgments}

This work is supported primarily by the de Beaumont Foundation with additional support from the Robert Wood Johnson Foundation, Robin Hood, and the New York State Health Foundation. The views presented here are those of the authors and should not be attributed to the de Beaumont Foundation, the Robert Wood Johnson Foundation, Robin Hood, the New York State Health Foundation or to their directors, officers, and staff.

\author{
*Sharon E. Perlman \\ E-mail: sperlma1@health.nyc.gov
}

